(C) 2018 IEEE. Personal use of this material is permitted. Permission from IEEE must be obtained for all other

uses, in any current or future media, including reprinting/republishing this material for advertising or promotional purposes, creating new collective works, for resale or redistribution to servers or lists, or reuse of any

copyrighted component of this work in other works.

\title{
SRLG-disjointness and geodiverse routing - a practical network study and operational conclusions
}

\author{
Boro Nedic*, Matthias Gunkel ${ }^{\dagger}$, Teresa Gomes ${ }^{\ddagger \S}$, Rita Girão-Silva ${ }^{\ddagger \S}$ \\ *Department of Mathematics and Natural Science, Darmstadt University of Applied Science, Germany \\ ${ }^{\dagger}$ Deutsche Telekom Technik GmbH - Optical Packet Transport, Darmstadt, Germany \\ ${ }^{\ddagger}$ Department of Electrical and Computer Engineering, University of Coimbra, Portugal \\ $\S$ Institute for Systems Engineering and Computers at Coimbra (INESC Coimbra), Portugal \\ boro.nedic@stud.h-da.de; Matthias.Gunkel@telekom.de; \{teresa,rita\}@deec.uc.pt
}

\begin{abstract}
The problem of disaster resilience is of paramount importance in today's telecommunication networks. Here, this problem is tackled by considering $1+1$ optical lightpath protection with maximally SRLG-disjoint paths and geodiversity in a transparent backbone network. Geodiversity was added to make the network more resilient to geographically correlated disasters. The aim is to estimate the increase of the path lengths (fibre lengths) and the increase in cost of the required transponders, with respect to simple link disjointness (no-SRLG nor geodiversity constraints) in a fully transparent optical network. The results obtained in a realistic test network show that SRLG-disjointness already ensures an acceptable geodiversity for the considered network.
\end{abstract}

Index Terms-SRLG-disjoint, min-sum, geodiverse routing, optical networks, disaster resilience

\section{INTRODUCTION}

The problem of disaster resilience is of paramount importance in today's telecommunication networks [1]. Hence, endto-end connectivity in backbone networks should be ensured against known risks of failure [2], [3]. A Shared Risk Link Group (SRLG) is a set of links that share a common risk of failure, such as fibres sharing a cable or a duct. Two paths that do not share a common risk of failure are said to be SRLG-disjoint. However, the determination of a pair of SRLGdisjoint paths is known to be a NP-complete problem [4]. Hence, in large networks and in the context of on-line routing, heuristics are used (see for example [5]-[7]) for obtaining SRLG-disjoint paths that seek to minimize some additive cost metric associated with the links of the paths. In general, one seeks to solve the min-sum problem, that is the sum of the cost of the paths, under SRLG-disjointness constraints.

SRLGs can also be used to represent geographically correlated failures. In [8], regional failures of a known radius are represented by the links affected by a circular disk. The authors show that the size of the list of SRLGs needed to cover all possible circular disk failures of a given radius was in practice only $20 \%$ larger than the total number of nodes in a network. In a closely related work [9] SRLGs, that represent

This article is based on work from COST Action CA15127. The work of T. Gomes and R. Girão-Silva has been partially supported by Fundação para a Ciência e a Tecnologia (FCT) under project grant UID/MULTI/00308/2013 and was financially supported by FEDER Funds and National Funds through FCT under project CENTRO-01-0145-FEDER-029312. disk failures affecting a fixed number of network nodes, are generated. The authors conclude that the number of such SRLGs is proportional to the product of the number of nodes in the networks multiplied by the fixed number of affected nodes.

The existence of SRLGs requests routing algorithms to find maximally SRLG-disjoint paths, when fully disjoint paths do not exist in the network [6], [10], [11]. The problem to be tackled in this work is to find two maximally SRLG-disjoint paths, which should always be link (or edge) disjoint, for all traffic demands directly guided over end-to-end optical lightpaths (that is, in a transparent optical network).

The need for routing taking into account geographically correlated failures is present in [12]-[14]. The concept of path diversification was introduced in [12], and it allows to use several paths selected using a measure for diversity and seeking to improve flow reliability. Using that concept, a routing protocol, that finds two paths at a certain distance, on the physical network topology, was proposed in [13]. To make a network more resilient to disasters, geodiverse paths are calculated in [14].

In this paper maximally SRLG-disjoint paths considering geodiversity in a backbone network will be calculated. The goal is to determine the increase of the path lengths (fibre lengths) and the increase in cost of the required transponders, with respect to simple link disjointness (no-SRLG nor geodiversity constraints).

After this Introduction section, the motivation for this work is further explained in Section II, followed by a formal description of the addressed problems in Section III. The resolution approach used for solving the formulated problems is in Section IV. Details on the calculation of the transponders' reach and cost are provided in Section $\mathrm{V}$ and results are presented in Section VI. Final remarks are given in Section VII.

\section{Motivation}

Considering the SRLGs in a network the problem is to find a maximally SRLG-disjoint path pair of min-sum cost for all traffic demands directly guided over end-to-end optical lightpaths. Here cost will be the actual fibre cable length, but it could be any additive cost. If the risk of failure is associated with the length of an SRLG, that is the length of duct or cable 
shared by the edges, then it is also relevant to obtain the minsum cost path pair with the minimal SRLG shared length.

The first objective is to observe how different are the shortest paths when SRLGs are not taken into account, that is if a min-sum cost path pair is used. This will allow to conclude how relevant are the inherent SRLGs from a topological point of view.

In order to increase the network resilience to geographically correlated failures, it was considered to obtain $D$-geodiverse path pairs, that is paths separated by $D \mathrm{~km}$ as defined in [14]. Hence, the second objective was to observe how geodiverse routing (for various values of $D$ ) would impact on the length of the paths, under SRLG-disjointness constraints.

The final objective of this work was an assessment of the cost in terms of required transponders, to satisfy a set of demands using coherent interfaces at $100 \mathrm{~Gb} / \mathrm{s}$ or beyond taking into account optical reach limitations [15]. As the fibre spectrum was modeled to be unconstrained, the lightpaths can always be guided over the calculated optimal path pair for each considered scenario. In order to keep the problem simple, the specific wavelength or spectrum assignment is not addressed.

The precise calculation of the distance between two paths requires detailed geographical information about each edge of the paths, which is not available. Hence given the coordinates of the nodes in the $x y$ plane, the edges will be assumed to follow the shortest lines connecting the corresponding end nodes. This will result in an optimistic calculation of the paths distances. Nevertheless, the cost of a path pair is the sum of the actual fibre length in kilometers of both paths, under SRLGdisjointness constraints.

Hence the final objective was to evaluate the increase in cost for simultaneous SRLG and geodiverse routing, to ensure that edges not sharing common risks are geographically distant.

\section{AdDRESSED PROBLEMS}

\section{A. Notation}

The physical network topology will be represented by $\mathcal{G}=$ $(V, E)$ where $V=\left\{v_{1}, v_{2}, \ldots, v_{|V|}\right\}$ is the set of $|V|$ nodes and $E=\left\{e_{1}, e_{2}, \ldots, e_{|E|}\right\}$ is the set of $|E|$ network links or edges (undirected arcs) represented by an unordered node pair $\left(e_{u}=\left\{v_{a}, v_{b}\right\}\right.$, with $\left.v_{a}, v_{b} \in V, u=1,2, \ldots,|E|\right)$.

In the Integer Linear Programming (ILP) models that will be formulated in Section IV, each edge from $E$ will be represented by two symmetrical (directed) arcs, so that edge $e_{u}=\left\{v_{a}, v_{b}\right\}$ will be represented by $a_{k}=\left(v_{a}, v_{b}\right)$ and $a_{k^{\prime}}=\left(v_{b}, v_{a}\right)$. The set of the directed arcs representing the links in $E$ will be denoted by $A$.

Let $\rho$ be the total number of risks that will affect more than one edge in the network. Set $Y$, with $Y=\left\{y_{1}, y_{2}, \ldots, y_{\rho}\right\}$ will denote the set of existing failure risks. If the event associated with risk $y_{r}$ occurs then a set of links, designated by $A_{r}$ will fail, i.e. $A_{r}$ is the set of arcs that defines the $r$-th SRLG. Note that one arc $a \in A$ may not belong to any $A_{r}, r=1, \ldots, \rho$, so $\cup_{r \in R} A_{r}$ may be only a subset of $A$.

Let $R$ be the set of indexes of all SRLGs in the network. For ease of notation, SRLG $A_{r}$ will be simply identified by $r$, with $r=1, \ldots, \rho$. Given the arc $a_{k}$, then $\phi_{k}$ is the set of indexes of SRLGs in $R$ which contain arc $a_{k}$, i.e. $r \in \phi_{k}$ if and only if $a_{k} \in A_{r}$.

A path is a continuous sequence of nodes (all different) from one source node, $s$, to a destination node $t,(s, t \in V)$, and is represented by $p=\left\langle s \equiv v_{1}, v_{2}, \ldots, v_{n} \equiv t\right\rangle$, where $\left(v_{i}, v_{i+1}\right) \in A, i \in\{1, \ldots, n-1\}$ and $n$ is the number of nodes in the path. Let $A_{p}$ be the set of arcs of path $p$ and $E_{p}$ the set of the corresponding edges. Let $\mathcal{P}_{s t}$ represent the set of all paths from $s$ to $t$ in the network. The set of path pairs, from $s$ to $t$, which are edge-disjoint is designated by $\mathcal{P}_{s t}^{2}$.

Let $\varphi_{p}^{r}$ be the set of arcs in path $p$ belonging to SRLG $r$, i.e. if $a_{k} \in \varphi_{p}^{r}$, then $a_{k} \in A_{r}$ and $a_{k} \in A_{p}$. The indexes of the union of all SRLGs of path $p$ is denoted by $R_{p}=\{r \in$ $\left.R: A_{r} \cap A_{p} \neq \emptyset\right\}$. The arcs that share an SRLG with the arcs of path $p$ are the arcs in $\cup_{r \in R_{p}} A_{r}$. The indexes of the SRLGs shared by path pair $(p, q)$ is represented by $R_{p, q}=R_{p} \cap R_{q}$.

The length of arc $a_{k} \in A$ is given by $l_{k}$, and the length of path $p$ will be denoted by $\bar{l}_{p}$, with $\bar{l}_{p}=\sum_{a_{k} \in A_{p}} l_{k}$. Given an SRLG $r$, the length of the arcs that may be affected by risk $y_{r}$ is designated by $l^{r}$. It corresponds to the overlapped length of the segments of the arcs in $A_{r}$ which can be affected by common risk $y_{r}$.

The length of the SRLGs shared by a path pair $(p, q)$ is given by $\tilde{l}_{p, q}$, defined as follows:

$$
\tilde{l}_{p, q}= \begin{cases}0 & \text { if } R_{p, q}=\emptyset \\ \sum_{r \in R_{p, q}} l^{r} & \text { otherwise }\end{cases}
$$

\section{B. D-geodiverse path pair}

In [14] the geographical distance between two paths is shown to depend only on the distance of the edges of the paths. Let $\delta\left(e_{u}, e_{w}\right)$ designate the (minimal) distance between two edges $e_{u}$ and $e_{w}$. The same notation will be used if arcs are considered instead of edges: $\delta\left(a_{j}, a_{k}\right)$ designates the distance between the edges corresponding to the arcs $a_{j}$ and $a_{k}$. The geodiversity value of path pair $(p, q) \in \mathcal{P}_{s t}^{2}$ is represented by $D_{p, q}$ and given by:

$$
D_{p, q}=\min _{e_{u} \in E_{p}, e_{w} \in E_{q}} \delta\left(e_{u}, e_{w}\right)
$$

When $e_{u}, e_{w}$ have a common end node, the distance $\delta\left(e_{u}, e_{w}\right)=0$, except if the common node is the source or the destination of the path pair, in which case $\delta\left(e_{u}, e_{w}\right)$ is calculated as in [14]. More specifically, the distance between two edges $e_{u}=\left(s, v_{1}\right)$ and $e_{w}=\left(s, v_{2}\right)$ is the minimum of two distances: the distance between node $v_{1}$ and edge $e_{w}$ and the distance between node $v_{2}$ and edge $e_{u}$ - and similarly for a pair of edges with node $t$ in common.

For each node pair $(s, t), D_{s t}^{M a x}=\max _{(p, q) \in P_{s, t}^{2}} D_{p, q}$ represents the maximal geographical distance that can be achieved for any path pair between $s$ and $t$. The value $D_{s t}^{M a x}$ (for all $(s, t)$ pairs) can be calculated in advance, so one can define, for a desired $D$-geodiversity:

$$
D_{s t}=\max \left(D, D_{s t}^{M a x}\right)
$$

We assume the networks are bi-connected, hence at least a node-disjoint pair of paths exists for every node pair in the 
network. By using value $D_{s t}$ when calculating a $D$-geodiverse path pair from $s$ to $t$, we ensure a solution will always exist for any $D>0$ in a bi-connected network, because we relax $D$ to each node pair maximal possible value $D_{s t}$.

\section{Addressed optimization problems}

The calculation of the minimum number of SRLGs in common, that is the number of SRLGs shared by a maximally disjoint path is an ancillary problem for obtaining among all maximally SRLG-disjoint path pairs the one of minimal total length. Formally, problem $\mathscr{P}_{1}$ seeks to obtain $\mu_{s t}$, the minimum number of SRLGs in common for all link-disjoint path pairs from node $s$ to node $t$ :

$$
\mu_{s t}=\min _{\left(p_{1}, p_{2}\right) \in \mathcal{P}_{s t}^{2}}\left|R_{p_{1}, p_{2}}\right|
$$

This problem is solved in [4].

Knowing $\mu_{s t}$, problem $\mathscr{P}_{2}$, which tackles the calculation of minimal total length among the maximally SRLG-disjoint path pairs (which are also link-disjoint), can be stated as:

$$
\lambda_{s t}=\min _{\left(p_{1}, p_{2}\right) \in \mathcal{P}_{s t}^{2}:\left|R_{p_{1}, p_{2}}\right|=\mu_{s t}}\left(\bar{l}_{p_{1}}+\bar{l}_{p_{2}}\right)
$$

A formulation for a similar problem can be found in [11].

The third problem $\left(\mathscr{P}_{3}\right)$, is again an auxiliary problem and consists in the calculation of the minimal total SRLG-length, among all link-disjoint path pairs:

$$
\tilde{\lambda}_{s t}=\min _{\left(p_{1}, p_{2}\right) \in \mathcal{P}_{s t}^{2}} \tilde{l}_{p_{1}, p_{2}}
$$

The fourth problem $\left(\mathscr{P}_{4}\right)$ is the minimization of the total length of a path pair, given that $\tilde{\lambda}_{s t}$ must be satisfied:

$$
\bar{\lambda}_{s t}=\min _{\left(p_{1}, p_{2}\right) \in \mathcal{P}_{s t}^{2}: \tilde{l}_{p_{1}, p_{2}}=\tilde{\lambda}_{s t}}\left(\bar{l}_{p_{1}}+\bar{l}_{p_{2}}\right)
$$

Finally, problem $\mathscr{P}_{5}$, the calculation of the minimal total length among all maximally SRLG-disjoint path pairs with geodiversity constraint $D_{s t}$, can be written as:

$$
\hat{\lambda}_{s t}=\min _{\left(p_{1}, p_{2}\right) \in \mathcal{P}_{s t}^{2}:\left|R_{p_{1}, p_{2}}\right|=\mu_{s t} \wedge D_{p_{1}, p_{2}} \geq D_{s t}}\left(\bar{l}_{p_{1}}+\bar{l}_{p_{2}}\right)
$$

\section{RESOLUTION APPROACH}

First the minimal number of shared SRLGs for each demand was calculated (resolution of problem $\mathscr{P}_{1}$ ). With these results, it was possible to calculate the minimum total length of the maximally SRLG-disjoint path pairs for the demands (thus solving problem $\mathscr{P}_{2}$ ).

The minimal length of shared SRLGs for each demand was obtained (resolution of problem $\mathscr{P}_{3}$ ) using the formulation in Subsection IV-D. This allowed to determine the minimum total length path pair, with minimal SRLG-length in common (resolution of problem $\mathscr{P}_{4}$ using the formulation in Subsection IV-E).

Finally geodiversity was considered, originating problem $\mathscr{P}_{5}$, which relies on the result obtained for problem $\mathscr{P}_{1}$ for each demand. To calculate the geodiversity one must obtain the geographical distance of each link to every other link and from each link to every node. As already mentioned, this was done assuming that each link goes in a straight line from node to node - this is a very optimistic way to calculate link distances.
One could have scaled up the obtained distance by some factor, or alternatively scale down the desired geodiversity. Therefore, the solution for a problem with a geodiversity of $D \mathrm{~km}$ will be optimistic in the sense that the real geodiversity is probably lower.

The objectives were to evaluate the increase in length and cost (due to the required transponders) when SRLGdisjointness and $D$-geodiversity was considered, with respect to link-disjoint paths. To solve the problems described above, several ILP formulations were used. The notation is introduced in the next subsection, followed by the used formulations.

\section{A. Additional notation}

For the formulation of the ILP problems, we will use the following indexes: $j, k=1, \ldots,|A|$ for identifying an arc $a_{j}$ or $a_{k} ; i=1, \ldots,|V|$ for identifying a node $v_{i} ; s$ and $t$ for identifying the source node and the destination node, respectively; $m=1,2$ for identifying if a path is the active one $(m=1)$ or the backup one $(m=2) ; r=1, \ldots, \rho$ for identifying the risks and the corresponding SRLGs. A path pair is identified by $\left(p_{1}, p_{2}\right)$. The indexes of the arcs leaving node $v_{i}$ are identified by $\mathcal{E}\left(i^{+}\right)$and the indexes of the arcs entering node $v_{i}$ are identified by $\mathcal{E}\left(i^{-}\right)$. Note that any arc $a_{k}$ leaving node $v_{i}$ will have a symmetrical arc $a_{k^{\prime}}$ entering the same node.

A set of parameters is calculated and known beforehand:

- $h_{r, k}$ indicates whether arc $a_{k}$ belongs to SRLG $r$ :

$$
h_{r, k}= \begin{cases}1 & \text { if } r \in \phi_{k} \\ 0 & \text { otherwise }\end{cases}
$$

As previously stated, $r \in \phi_{k}$ is equivalent to $a_{k} \in A_{r}$.

- $D_{s t}$, as calculated in equation (3).

- $d_{k, j}$ indicates whether arcs $a_{k}$ and $a_{j}$ are geodiverse (for a connection from $s$ to $t$ ):

$$
d_{k, j}= \begin{cases}1 & \text { if } \delta\left(a_{k}, a_{j}\right)<D_{s t} \\ 0 & \text { otherwise }\end{cases}
$$

- the length of the arcs $l_{k}$ and the overlapped length of the segments in SRLG $r$, i.e. $l^{r}$.

The binary decision variables used in the formulation are:

- $x_{k, m}$ indicates whether arc $a_{k}$ belongs to path $p_{m}$ :

$$
x_{k, m}= \begin{cases}1 & \text { if } a_{k} \in p_{m} \\ 0 & \text { otherwise }\end{cases}
$$

- $z_{r, m}$ indicates whether SRLG $r$ affects an arc of path $p_{m}$ :

$$
z_{r, m}= \begin{cases}1 & \text { if } r \in R_{p_{m}} \\ 0 & \text { otherwise }\end{cases}
$$

- $g_{r}$ indicates whether SRLG $r$ includes arcs belonging simultaneously to both paths:

$$
g_{r}= \begin{cases}1 & \text { if } \exists a_{j} \in \varphi_{p_{1}}^{r} \wedge \exists a_{k} \in \varphi_{p_{2}}^{r} \\ 0 & \text { otherwise }\end{cases}
$$

For all the formulated problems, the arcs forming the solution path pair $\left(p_{1}^{*}, p_{2}^{*}\right)$ are given in the decision variables $x_{k, 1}$ and $x_{k, 2}$.

\section{B. Minimizing the number of SRLGs in common}

Problem $\mathscr{P}_{1}$ was solved using the formulation proposed in [4]. We present the formulation here for a demand originat- 
ing in node $s$ and terminating in node $t$, as the formulations of the other problems include some of the constraints of $\mathscr{P}_{1}$.

$$
\min \sum_{r=1}^{\rho} g_{r}
$$

subject to:

$$
\begin{aligned}
& \sum_{k \in \mathcal{E}\left(i^{+}\right)} x_{k, m}-\sum_{k \in \mathcal{E}\left(i^{-}\right)} x_{k, m}=\left\{\begin{aligned}
1 & \text { if } v_{i}=s \\
-1 & \text { if } v_{i}=t \\
0 & \text { otherwise }
\end{aligned}\right. \\
& \forall i=1, \ldots,|V| ; m=1,2 \\
& x_{k, 1}+x_{k, 2} \leq 1, \quad \forall k=1, \ldots,|A| \\
& x_{k, 1}+x_{k^{\prime}, 2} \leq 1, \quad \forall k=1, \ldots,|A| \\
& x_{k, m}+x_{k^{\prime}, m} \leq 1, \quad \forall k=1, \ldots,|A| ; m=1,2 \\
& \sum_{k \in \mathcal{E}\left(i^{+}\right)} x_{k, m}+\sum_{k \in \mathcal{E}\left(i^{-}\right)} x_{k, m} \leq 2 \\
& \forall i=1, \ldots,|V| ; m=1,2 \\
& \sum_{k=1}^{|A|} h_{r, k} x_{k, m} \leq|A| z_{r, m}, \quad \forall r=1, \ldots, \rho ; m=1,2 \\
& z_{r, 1}+z_{r, 2}-g_{r} \leq 1, \quad \forall r=1, \ldots, \rho \\
& \text { binary } x_{k, m}, z_{r, m}, g_{r} \\
& \forall k=1, \ldots,|A| ; m=1,2 ; r=1, \ldots, \rho
\end{aligned}
$$

Constraints (9) are the usual flow conservation constraints. Constraints (10)-(11) guarantee that an arc (or an arc and its symmetrical) cannot be used in both paths simultaneously. Constraints (12)-(13) are formulated to guarantee that no cycles (or loops) are formed. An arc and its symmetrical cannot be used in the same path; any node in either path cannot have more than two of its arcs in that path, which prevents certain configurations of cycles from appearing.

Constraint (14) allows to know if an SRLG affects an arc of a path. On the one hand, if at least an arc $a_{k}$ belonging to SRLG $r$ (i.e. $h_{r, k}=1$ ) is used in path $p_{m}$ (i.e. $x_{k, m}=1$ ) then the constraint is satisfied only if $z_{r, m}=1$; on the other hand, if none of the arcs in path $p_{m}$ is affected by risk $r$, then the left-hand side of the inequation is 0 , which means that $z_{r, m}$ may be 0 or 1 . However, the constraint (15) and the fact that we are minimizing the number of variables $g_{r}$ which are 1 , will lead to $z_{r, m}=0$. In fact, a variable $g_{r}$ will be 1 only if $z_{r, 1}$ and $z_{r, 2}$ are simultaneously 1 (i.e. both paths have arcs affected by the risk $r$ ), as guaranteed by constraint (15) and the considered objective function.

\section{Minimizing the path-length of a link-disjoint path pair with minimal number of SRLGs in common}

Problem $\mathscr{P}_{2}$ was solved using a formulation similar to the one in [11]. For completeness of the paper, we present the formulation here for a demand originating in node $s$ and terminating in node $t$.

$$
\min \sum_{k=1}^{|A|}\left(x_{k, 1} l_{k}+x_{k, 2} l_{k}\right)
$$

subject to: constraints (9)-(11), (14)-(16) and

$$
\mu_{s t}=\sum_{r=1}^{\rho} g_{r}
$$

where $\mu_{s t}$ is the value obtained as a result of the resolution of $\mathscr{P}_{1}$. A lexicographic optimization approach is used, as this problem is solved after having found a solution for $\mathscr{P}_{1}$.

In the formulation of this problem, constraints (12) and (13) may be omitted, as the objective function of $\mathscr{P}_{2}$ naturally leads to the avoidance of any superfluous links in the solution.

\section{Minimizing the length of SRLGs in common}

Problem $\mathscr{P}_{3}$ tackles the minimization of the length of the common SRLGs in an edge-disjoint path pair for a demand originating in node $s$ and terminating in node $t$.

$$
\min \sum_{r=1}^{\rho} g_{r} l^{r}
$$

subject to: constraints (9)-(16).

\section{E. Minimizing the path-length of a link-disjoint path pair with minimal length of SRLGs in common}

Problem $\mathscr{P}_{4}$ deals with the minimization of the length of the edge-disjoint path pair for a demand originating in node $s$ and terminating in node $t$, considering that the common SRLGs have minimal length.

$$
\min \sum_{k=1}^{|A|}\left(x_{k, 1} l_{k}+x_{k, 2} l_{k}\right)
$$

subject to: constraints (9)-(11), (14)-(16) and

$$
\tilde{\lambda}_{s t}=\sum_{r=1}^{\rho} g_{r} l^{r}
$$

where $\tilde{\lambda}_{s t}$ is the value obtained as a result of the resolution of $\mathscr{P}_{3}$. A lexicographic optimization approach is used, as this problem is solved after having found a solution for problem $\mathscr{P}_{3}$.

F. Minimizing the total path-length of a path pair, under (maximal) SRLG-disjointness and geodiversity constraints

This problem requires the calculation of the maximum geodiversity that can be achieved between every node pair of interest for a demand originating in node $s$ and terminating in node $t$. A formulation for solving this problem can be found in [14].

Problem $\mathscr{P}_{5}$ is similar to problem $\mathscr{P}_{2}$, but now a constraint on the geodiversity is considered.

$$
\min \sum_{k=1}^{|A|}\left(x_{k, 1} l_{k}+x_{k, 2} l_{k}\right)
$$

subject to: constraints (9), (14)-(17) and

$$
\begin{aligned}
& \sum_{k \in \mathcal{E}\left(i^{+}\right)}^{x_{(s, t), 1}+x_{(s, t), 2} \leq 1}\left(x_{k, 1}+x_{k, 2}\right) \leq 1, \\
& \quad \forall i=1, \ldots,|V|: v_{i} \neq s \wedge v_{i} \neq t \\
& \left(x_{k, 1}+x_{j, 2}\right) d_{k, j} \leq 1, \forall k, j=1, \ldots,|A|
\end{aligned}
$$


A lexicographic optimization approach is used, as this problem is solved after having found a solution for problem $\mathscr{P}_{1}$.

In the formulation of this problem, constraints (10) and (11) may be omitted, because we added the node disjointness constraint (20). Due to the removal of (10) and (11), constraint (19) had to be added, to guarantee that the direct arc between $s$ and $t$ (if it exists) is not used in both paths simultaneously.

Constraint (21) guarantees the geodiversity. On the one hand, if $d_{k, j}=0$ then the constraint is always satisfied, which means that the arcs $a_{k}$ and $a_{j}$ may be freely used in the paths, as they are geodiverse; on the other hand, if $d_{k, j}=1$ then the constraint is satisfied only if the $\operatorname{arcs} a_{k}$ and $a_{j}$ are not used in both paths, as they are not geodiverse.

\section{CALCUlation OF TRANSPONDERS' REACH AND COST}

Given a traffic matrix with demands in $\mathrm{Gb} / \mathrm{s}$, using the models in the previous section one can solve problems $\mathscr{P}_{2}$, $\mathscr{P}_{4}$ and $\mathscr{P}_{5}$, to obtain the optimal path pairs for those demands. To calculate the cost of each solution in terms of required Reconfigurable Optical Add-Drop Multiplexers (ROADMs) one must take into account the fibre length of the paths. This will determine the optical reach and the maximum rate of each considered transponder.

\section{A. Transponder reach}

The maximum reach is achieved if the Bit Error Rate (BER) exceeds a certain threshold. This is usually related to minimum Optical Signal-to-Noise Ratio (OSNR). In order to take into account both, the noise contribution from Amplified Spontaneous Emission (ASE) and Non-Linear Interference (NLI) due to the Kerr effect in the fibre, we utilized the generalized Gaussian noise model according to [15]:

$$
\text { OSNR }=\frac{P_{T x, c h}}{P_{\mathrm{ASE}}+P_{\mathrm{NLI}}}
$$

where $P_{T x, c h}$ is the input power per channel $(c h)$ of the transmitter $(T x)$.

The ASE noise power is given by [15]:

$$
P_{\mathrm{ASE}}=\left[N_{s}(G-1) F h \nu\right] B_{n}
$$

with $B_{n}$ the bandwidth at which the noise is measured, $N_{s}$ the number of amplifier spans, $G$ the performance gain of the Erbium Doped fibre Amplifier (EDFA), $F$ the EDFA's noise figure, $h$ the Planck effect quantum, and $\nu$ the frequency.

The noise power due to NLI is given by [15]:

$$
\begin{aligned}
P_{\mathrm{NLI}} & =\left(\frac{2}{3}\right)^{3} N_{s} B_{n} \psi \\
\psi & =\gamma^{2} L_{e f f} P_{T x, c h}^{3} \frac{\log _{2}\left(\pi^{2}\left|\beta_{2}\right| L_{e f f} N_{c h}^{2} R_{s}^{2}\right)}{\pi\left|\beta_{2}\right| R_{s}^{3}}
\end{aligned}
$$

with $\gamma$ the fibre nonlinearity coefficient, $L_{\text {eff }}$ the effective fibre length, $N_{c h}$ the number of channels, $R_{s}$ the baud rate and $\beta_{2}$ the fibre dispersion (for more details see [15]).

\begin{tabular}{|c|c|c|c|c|c|c|}
\hline \multirow{2}{*}{ Number of carriers } & \multicolumn{2}{|c|}{ Carrier } & 1 & 2 & \multirow{2}{*}{\multicolumn{2}{|c|}{$\begin{array}{l}4 \\
2.56\end{array}$}} \\
\hline & Fact & & 1 & 1.6 & & \\
\hline \multirow{2}{*}{ Maximal baudrate } & Gbaud & 34 & 4 & & 52 & 65 \\
\hline & Factor & 1 & 1. & .1 & 1.2 & 1.3 \\
\hline Adaptability of capacity & \multicolumn{6}{|c|}{$\frac{C_{\max }}{C_{\min }} 0.525^{\log _{2}\left(\frac{C_{\max }}{C_{\min }}\right)}$} \\
\hline
\end{tabular}

Replacing $P_{\mathrm{ASE}}$ and $P_{\mathrm{NLI}}$ in equation (22) by the expressions in equations (23) and (24), respectively, and then solving
TABLE I: Factors that influence the cost of transponders

the resulting equation in order to get $N_{s}$, one can obtain the maximum number of spans for the considered minimal OSNR:

$$
N_{s_{\text {max }}}=\frac{P_{T x, c h}}{\mathrm{OSNR}_{\text {min }} \cdot B_{n}\left((G-1) F h \nu+\left(\frac{2}{3}\right)^{3} \psi\right)}
$$

The optical reach of a lightpath is given by $N_{s_{\max }} L_{s}$, where $L_{s}$ is the length of the span.

\section{B. Transponder cost}

We introduce normalized transponder costs. The reference, i.e. $100 \%$ cost, is a state-of-the-art $100 \mathrm{~Gb} / \mathrm{s}$ coherent transponder implemented by a single carrier with DualPolarization Quadrature Phase-Shift Keying (DP-QPSK) and roughly 34 Gbaud electronic speed.

The costs of more advanced transponders are assumed to mainly depend on three factors. The first is the number of optical carriers (lasers). For example, we assume a transponder composed of two carriers is cheaper than two individual transponders with a single carrier each. Hence, it is always more cost-efficient to choose a transponder with two or more carriers than choosing two or more transponders with a single carrier. The second factor is the maximum baudrate. We assume that the baudrate contributes only moderately to the overall cost of a transponder. Doubling the maximal baudrate means that the cost increases roughly one-third. The last contribution to cost scaling is the adaptability of the transponder capacity, meaning that a fixed transponder which supports a single modulation format and thus a single capacity only is cheaper than a modulation-flexible transponder supporting multiple modulation formats and related capacities. For example, DP-QPSK-only modulation is cheaper than the adaptable support of DP-QPSK, DP-8QAM (Quadrature Amplitude Modulation) and DP-16QAM modulations which means that the capacity can be flexibly doubled. In this sense, $C_{\max }$ and $C_{\min }$ refer to the maximum and minimum capacity induced by modulation adaptivity. The respective formula in Table I was inspired by [16, eqs. (33)-(34)], tailored so that doubling the capacity comes along with $5 \%$ extra costs.

\section{Results}

Results were obtained for a generic sample of the backbone network of Deutsche Telekom (DT) with 12 nodes (see Figure 1). We start by presenting, in subsection VI-A, results for data provided by DT. As details of these data cannot be made public, we also present, in subsection VI-B, results for synthetic data, based on the original information provided by DT, by introducing a certain degree of randomness. 


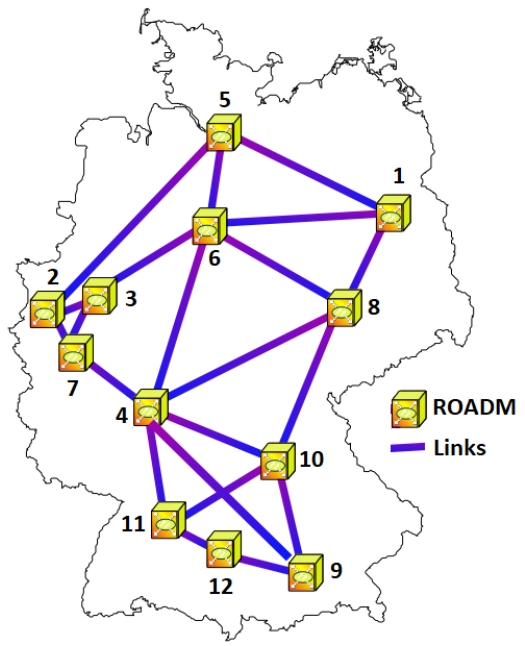

Fig. 1: Generic backbone network of Deutsche Telekom

The problems described in Sections III and IV were solved using CPLEX 12.6 [17]. For each demand, an edge-disjoint path pair was obtained that led to the solution with: (i) minsum of the length of the path pair - "Basic"; (ii) minimal number of SRLGs in common, which corresponds to problem $\mathscr{P}_{2}$ - "min \#SRLG"; (iii) minimal length of SRLGs in common, which corresponds to problem $\mathscr{P}_{4}$ - "min SRLG-length"; (iv) minimal total path length, considering SRLG-disjoint path pairs and geodiversity constraints, with a distance $D$, which corresponds to problem $\mathscr{P}_{5}-$ "D= $x \mathrm{~km}$ " for $D=x \mathrm{~km}$ and "D=Dmax" for the maximal possible distance such that geodiverse path pairs may still be found.

The data used for the $D$-geodiverse routing, i.e. the link-tolink, link-to-node and $D_{s t}$ (for all $(s, t)$ node pair) distances, based on links following the shortest path in a plane can be found in [18].

For the cost analysis, the parameters used to calculate the transponder optical reach (see equation (26)) were: $N_{c h}=$ 96; $\gamma=1.37\left[(\mathrm{~W} . \mathrm{km})^{-1}\right] ; L_{\text {eff }}=\left(1-e^{-2 \alpha L_{s}}\right) /(2 \alpha) ; \alpha=$ $0.22[\mathrm{~dB} / \mathrm{km}] ; L_{s}=80[\mathrm{~km}] ; \beta_{2}=-D \lambda^{2} /(2 \pi c)\left[\mathrm{ps}^{2} / \mathrm{km}\right] ;$ $D=17[\mathrm{ps} /(\mathrm{km} \cdot \mathrm{nm})]$, and $c$ the light velocity in free space. Additionally it was considered an OSNR penalty of 4 [dB] which accounts for ageing, implementation imperfections, and additional imperfections inside the fibre infrastructure.

It was assumed that 7 types of transponders with different properties (cost, adaptable capacity and reach) could be used. The reach and cost of the selected transponder was calculated as explained in Section V.

The cheapest transponder capable of transporting the traffic of a demand (both in the active and the backup path note that the latter is always the longest path) was always chosen. If a single transponder cannot support the necessary reach and traffic volume, the traffic must be split and more transponders will be required in a fully transparent optical network. Note that this corresponds to routing without fibre capacity constraints.

\section{A. Results for data provided by DT}

The traffic matrix, the length of the edges (in $\mathrm{km}$ ), the SRLGs and their corresponding fibre lengths (in $\mathrm{km}$ ) for the generic network in Figure 1 were provided by DT. For the length of the edges, the actual fibre length in $\mathrm{km}$ was used, not the air lines between the ROADM locations.

Results for the problems in Sections III-IV are in Figure 2. In these results, physical constraints (optical reach) were ignored. In Figure 2a, the total length of the paths for all the demands is presented, for the different problems. In Figure $2 b$, the length variation of the paths is displayed, in relation to the value obtained with the so-called "Basic" solution. Note that for node 2 (as source or destination) only maximally SRLGdisjoint path pairs can be obtained: SRLG 6 , or 7 , or 8 will be shared - see last column of Table II.

The solutions concerning the length of the active paths are very similar for all the problems. As for the solutions for the backup paths and also for both paths, the variation is much more clear. If the objective is to have minimum length of common SRLGs, the paths are longer than when minimizing the number of SRLGs in common. That is why only the minimal number of SRLGs in common was considered when including geodiversity constraints (problem $\mathscr{P}_{5}$ ).

The results show that SRLG-disjointness already ensures a good geodiversity, as the increase in total path length from "min \#SRLG" to " $D=50 \mathrm{~km}$ " and " $\mathrm{D}=100 \mathrm{~km}$ " is very small. For increasing values of $D$, the total path length increases from $15.8 \%$ to $26.0 \%$ compared with the "Basic" solution. For higher values of $D$, the backup paths have to be longer, so that a higher geodiversity is obtained.

For demands where the source and destination are far away, there is not a remarkable difference between link disjointness and SRLG-disjointness with geodiversity, hence the fibre length increase is mostly due to demands where the source and destination are relatively close.

The obtained results for the costs are displayed in Figure 3. In Figure 3a, the total cost of the coherent transponders for all the demands is displayed in arbitrary units ([a.u.]), and in Figure $3 \mathrm{~b}$ the relative cost increase with respect to the "Basic" solution is presented. One of the parameters that influences the cost of transponders is the length of the paths. Comparing the results in Figures $2 b$ and $3 b$, the variation of the length of the backup path and the variation of the cost of the transponders follow a similar trend, i.e. when longer paths are considered, the cost of the needed transponders increases. However, this increase is not in the same proportion: the increase in backup path length ranges from $21.3 \%$ to $41.6 \%$, whereas the increase in transponders costs ranges from $7.0 \%$ to $10.3 \%$. Finally, it should be pointed out that only a few node pairs require superior transponders (from 5 node pairs in "min \#SRLG" to 9 for "D=Dmax"), with respect to the "Basic" solution.

\section{B. Results for synthetic data}

The same generic sample of the backbone network of DT (Figure 1) is used in these calculations. The synthetic data used in this study are displayed in Tables II-III. All the lengths are 


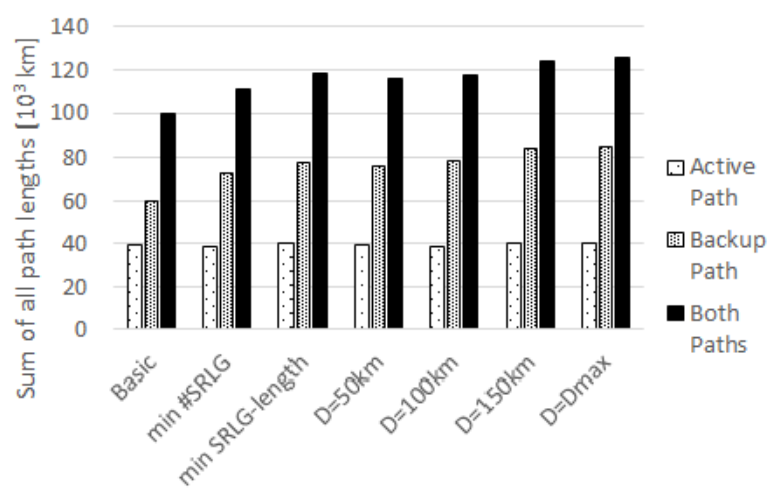

(a) Total path length

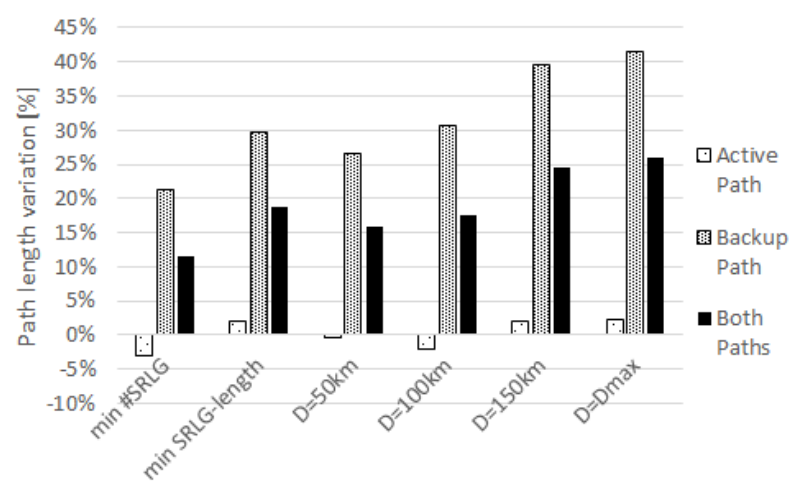

(b) Path length variation (to "Basic")

Fig. 2: Results for the sum of the length of all the paths

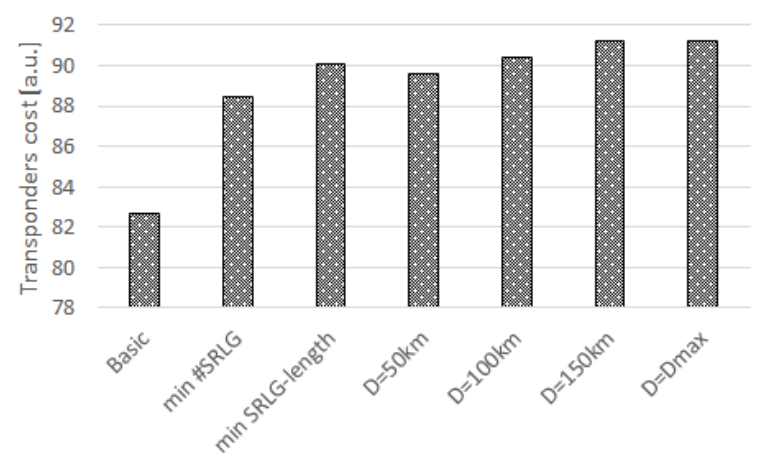

(a) Total cost of transponders

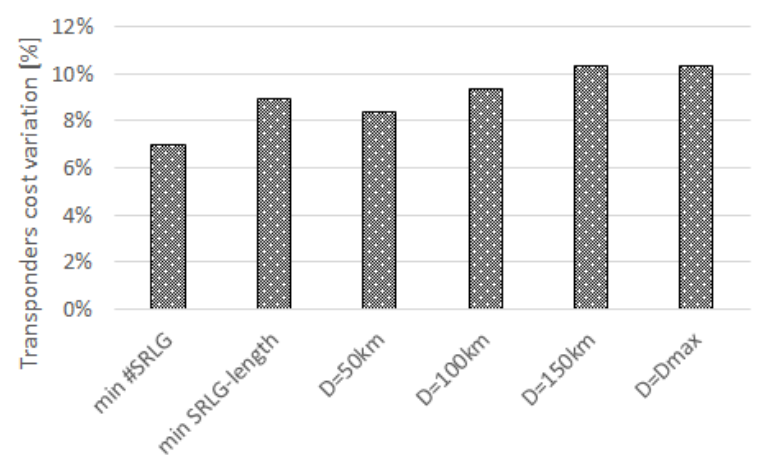

(b) Transponders cost variation (to "Basic")

Fig. 3: Results for the costs of the transponders

integer values in $\mathrm{km}$. The length of the edges (see Table II) was calculated based on the Euclidean distance between the network nodes. A correction factor was employed so that the total length of all the edges in this study was close to the total length of the edges in the data used in Subsection VI-A, and finally a random term was applied to each value. The SLRGs are defined by common ducts and/or cables shared by the fibres. The length of such common segments are shown in Table III. They are a percentage of the minimal length of the edges that are part of the SRLG. For the calculation of cost of the transponders, the traffic matrix (integer values in $\mathrm{Gb} / \mathrm{s}$ ) in Table IV was used.

The total length of the paths and their variation with respect to the "Basic" solution are displayed in Table V for all the demands, for the different problems formulated in Sections IIIIV. The results for the cost of the transponders are presented in Table VI.

The results for the synthetic data follow the same pattern as the ones for the DT data. Regarding the length of the paths, the variation of the backup path is larger than for the active path, as previously noted. The variation range is similar, but slightly smaller than the one observed with the DT data set and affecting a smaller number of traffic demands. This is reflected in a smaller increase of the cost of transponders for the synthetic data case, with respect to the corresponding "Basic" solution. Similarly to what was observed in Section VI-A, only a few node pairs require superior transponders (for example, from 3 node pairs in "min \#SRLG" to 7 for " $D=D m a x ")$, with respect to the "Basic" solution.

\section{CONCLUSION}

The problem of determining maximally SRLG-disjoint paths with geodiversity constraints was introduced, formalized and solved. The objective was to evaluate the increase in fibre length and equipment cost compared to $1+1$ protection.

To determine the cost increase, due to the longer path distances required by maximally SRLG-disjointness and geodiverse routing, the reach of the transponders was calculated and an estimate of the cost of the transponders, with respect to a reference transponder was proposed. Results were presented for a generic sample of the transparent optical backbone network of DT (with two data sets). For the considered network, it was observed that SRLG-disjointness already ensures an acceptable geodiversity for most of the node pairs. When fully SRLG-disjoint path pairs could not be obtained, it was verified that minimizing the length of the SRLGs in common led to excessively longer routes. Regarding cost increase it 
TABLE II: Links fibre length in $\mathrm{km}$ and corresponding SRLGs

\begin{tabular}{|r|r|r|l|}
\hline \#node & \#node & fibre length [km] & SRLG number (from DT) \\
\hline 1 & 5 & 440 & \\
1 & 6 & 473 & 2,3 \\
1 & 8 & 271 & \\
2 & 3 & 98 & 6,8 \\
2 & 5 & 614 & $1,5,7,8$ \\
2 & 7 & 58 & $6,7,9$ \\
3 & 6 & 283 & 4,5 \\
3 & 7 & 153 & 2 \\
4 & 6 & 426 & \\
4 & 7 & 242 & 9 \\
4 & 8 & 457 & $10,11,13$ \\
4 & 9 & 559 & $10,12,14,17,18$ \\
4 & 10 & 337 & 10,11 \\
4 & 11 & 279 & 10,12 \\
5 & 6 & 209 & 1,4 \\
6 & 8 & 343 & 2,3 \\
8 & 10 & 396 & 13 \\
9 & 10 & 265 & 15,18 \\
9 & 12 & 228 & \\
10 & 11 & 313 & $14,15,16$ \\
11 & 12 & 128 & 16,17 \\
\hline
\end{tabular}

TABLE III: SRLG fibre length in kilometers

\begin{tabular}{|r|r|r|r|r|r|r|r|r|r|r|r|r|r|r|r|r|r|r|}
\hline SRLG & 1 & 2 & 3 & 4 & 5 & 6 & 7 & 8 & 9 & 10 & 11 & 12 & 13 & 14 & 15 & 16 & 17 & 18 \\
\hline Length & 31 & 18 & 47 & 29 & 25 & 12 & 9 & 10 & 9 & 24 & 44 & 53 & 27 & 35 & 37 & 17 & 17 & 35 \\
\hline
\end{tabular}

TABLE IV: Traffic matrix (maximum of the demands in both directions) in $\mathrm{Gb} / \mathrm{s}$

\begin{tabular}{|r|rrrrrrrrrrr|}
\hline \#node & 1 & 3 & 4 & 5 & 6 & 7 & 8 & 9 & 10 & 11 & 12 \\
\hline 1 & 57 & 86 & 366 & 89 & 89 & 76 & 22 & 33 & 18 & 38 & 91 \\
2 & & 265 & 179 & 131 & 261 & 182 & 76 & 41 & 31 & 78 & 64 \\
3 & & & 235 & 91 & 87 & 96 & 97 & 25 & 77 & 5 & 67 \\
4 & & & & 585 & 202 & 197 & 455 & 627 & 514 & 617 & 179 \\
5 & & & & & 7 & 47 & 63 & 55 & 92 & 49 & 14 \\
6 & & & & & & 4 & 87 & 59 & 74 & 95 & 46 \\
7 & & & & & & & 73 & 82 & 92 & 53 & 10 \\
8 & & & & & & & & 29 & 77 & 69 & 88 \\
9 & & & & & & & & & 30 & 7 & 36 \\
10 & & & & & & & & & & 58 & 60 \\
11 & & & & & & & & & & & 11 \\
\hline
\end{tabular}

TABLE V: Sum of the total length of the paths for all the demands $[\mathrm{km}]$ (in brackets the relative variation of total path lengths in comparison with the "Basic" solution)

\begin{tabular}{|l|cc|cc|cc|}
\hline Solution & \multicolumn{2}{|c|}{ Active Path } & \multicolumn{2}{c|}{ Backup Path } & \multicolumn{2}{c|}{ Both Paths } \\
\hline Basic & 39809 & & 60434 & & 100243 & \\
min \#SRLG & 39223 & $(-1.47 \%)$ & 70170 & $(16.11 \%)$ & 109393 & $(9.13 \%)$ \\
min SRLG-length & 39912 & $(0.26 \%)$ & 74815 & $(23.80 \%)$ & 114727 & $(14.45 \%)$ \\
D=50km & 39223 & $(-1.47 \%)$ & 72561 & $(20.07 \%)$ & 111784 & $(11.51 \%)$ \\
D=100km & 39613 & $(-0.49 \%)$ & 76702 & $(26.92 \%)$ & 116315 & $(16.03 \%)$ \\
D=150km & 40812 & $(2.52 \%)$ & 80987 & $(34.01 \%)$ & 121799 & $(21.50 \%)$ \\
D=Dmax & 41088 & $(3.21 \%)$ & 82646 & $(36.75 \%)$ & 123734 & $(23.43 \%)$ \\
\hline
\end{tabular}

TABLE VI: Sum of the total cost of the transponders for the longest path for all the demands [a.u.] (in brackets the relative variation of total cost in comparison with the "Basic" solution)

\begin{tabular}{|l|cc|}
\hline Solution & \multicolumn{2}{|c|}{ cost [a.u.] } \\
\hline Basic & 84.04 & \\
min \#SRLG & 86.18 & $(2.55 \%)$ \\
min SRLG-length & 87.53 & $(4.15 \%)$ \\
D=50km & 87.32 & $(3.90 \%)$ \\
D=100km & 88.13 & $(4.87 \%)$ \\
D=150km & 88.13 & $(4.87 \%)$ \\
D=Dmax & 88.95 & $(5.84 \%)$ \\
\hline
\end{tabular}

was concluded that only a few node pairs require additional investment. Generally, we can conclude that tackling SRLGdisjointness of lightpaths requires an extra cost between 7.0\% and $8.9 \%$ (or $2.6 \%-4.2 \%$ with the synthetic data); from there including geodiversity in addition means only a further maximum value of $3.3 \%$ on top.

Regarding future work, other network topologies should be considered to verify if the main conclusions from the tested network still hold. One could also assume more advanced high-capacity transponder types offered in the near future.

\section{REFERENCES}

[1] J. Rak, D. Hutchison, E. Calle, T. Gomes, M. Gunkel, P. Smith, J. Tapolcai, S. Verbrugge, and L. Wosinska, "Recodis: Resilient communication services protecting end-user applications from disasterbased failures," in 18th Int. Conf. Transparent Optical Networks (ICTON 2016).

[2] T. Gomes, J. Tapolcai, C. Esposito, D. Hutchison, F. Kuipers, J. Rak, A. de Sousa, A. Iossifides, R. Travanca, J. André, L. Jorge, L. Martins, P. O. Ugalde, A. Pašić, D. Pezaros, S. Jouet, S. Secci, and M. Tornatore, "A survey of strategies for communication networks to protect against large-scale natural disasters," in 8th Int. Workshop Resilient Networks Design and Modeling (RNDM 2016) pp. 11-22.

[3] A. Mauthe, D. Hutchison, E. K. Cetinkaya, I. Ganchev, J. Rak, J. P. G. Sterbenz, M. Gunkel, P. Smith, and T. Gomes, "Disaster-resilient communication networks: Principles and best practices," in 8th Int. Workshop Resilient Networks Design and Modeling (RNDM 2016), pp. $1-10$.

[4] J. Q. Hu, "Diverse routing in optical mesh networks," IEEE Trans. Commun., vol. 51, no. 3, pp. 489-494, March 2003.

[5] A. Todimala and B. Ramamurthy, "IMSH: An iterative heuristic for SRLG diverse routing in WDM mesh networks," in 13th Int. Conf. Computer Communications and Networks, ICCCN'2004, pp. 199-204.

[6] M. J. Rostami, S. Khorsandi, and A. A. Khodaparast, "CoSE: A SRLG-disjoint routing algorithm," in Proc. Fourth Eur. Conf. Universal Multiservice Networks (ECUMN'07), 2007.

[7] T. Gomes, C. Simões, and L. Fernandes, "Resilient routing in optical networks using SRLG-disjoint path pairs of min-sum cost," Telecommun. Syst., vol. 52, no. 2, pp. 737-749, 2013.

[8] J. Tapolcai, L. Rónyai, B. Vass, and L. Gyimóthi, "List of shared risk link groups representing regional failures with limited size," in IEEE INFOCOM 2017 - IEEE Conf. Computer Communications, pp. 1-9.

[9] B. Vass, E. Berczi-Kovacs, and J. Tapolcai, "Enumerating shared risk link groups of circular disk failures hitting $k$ nodes," in 13th Int. Conf. Design of Reliable Communication Networks (DRCN 2017), pp. 1-9.

[10] M. J. Rostami, A. A. E. Zarandi, and S. M. Hoseininasab, "MSDP with ACO: A maximal SRLG disjoint routing algorithm based on ant colony optimization," J. Netw. Comput. Appl., vol. 35, pp. 394-402, 2012.

[11] T. Gomes, L. Jorge, P. Melo, and R. Girão-Silva, "Maximally node and SRLG-disjoint path pair of min-sum cost in GMPLS networks: a lexicographic approach," Photonic Netw. Commun., vol. 31, no. 1, pp. 11-22, 2016.

[12] J. P. Rohrer, A. Jabbar, and J. P. G. Sterbenz, "Path diversification for future internet end-to-end resilience and survivability," Telecommun. Syst., vol. 56, no. 1, pp. 49-67, 2014.

[13] Y. Cheng, J. Li, and J. P. G. Sterbenz, "Path geo-diversification: Design and analysis," in 5th Int. Congr. Ultra Modern Telecommunications and Control Systems and Workshops (ICUMT), 2013, pp. 46-53.

[14] A. de Sousa, D. Santos, and P. Monteiro, "Determination of the minimum cost pair of D-geodiverse paths," in 13th Int. Conf. Design of Reliable Communication Networks (DRCN 2017), pp. 1-8.

[15] P. Poggiolini, A. Carena, V. Curri, G. Bosco, and F. Forghieri, "Analytical modeling of nonlinear propagation in uncompensated optical transmission links," IEEE Photonics Tech. L., vol. 23, no. 11, pp. 742-744, June 2011.

[16] A. Eira, M. Quagliotti, and J. Pedro, "Impact of client- and line-side flexibility in the lifecycle of next-generation transport networks [invited],"J. Opt. Commun. Netw., vol. 8, no. 7, pp. A101-A115, 2016.

[17] IBM ILOG CPLEX Optimization Studio V12.6. IBM, 2013.

[18] http://www.deec.uc.pt/ /teresa/rndm2018, June 2018 